\title{
The sub-energetic GRB 031203 as a cosmic analogue to GRB 980425
}

\author{
A. M. Soderberg ${ }^{1}$, S. R. Kulkarni ${ }^{1}$, E. Berger ${ }^{1}$, D. W. Fox ${ }^{1}$, M. Sako $^{2}$, \\ D. A. Frail ${ }^{3}$, A. Gal-Yam ${ }^{1}$, D. S. Moon ${ }^{4}$, S. B. Cenko ${ }^{4}$, S. A. Yost ${ }^{4}$, \\ M. M. Phillips ${ }^{5}$, S. E. Persson ${ }^{5}$, W. L. Freedman ${ }^{5}$, P. Wyatt ${ }^{5}$, \\ R. Jayawardhana ${ }^{6} \&$ D. Paulson ${ }^{6}$ \\ ${ }^{1}$ Caltech Optical Observatories 105-24, California Institute of Technology, Pasadena, CA 91125, USA \\ ${ }^{2}$ Stanford Linear Accelerator Center, 2575 Sand Hill Road M/S 29, Menlo Park, CA 94025, USA \\ 3 National Radio Astronomy Observatory, P.O. Box 0, Socorro, New Mexico 87801, USA \\ ${ }^{4}$ Space Radiation Laboratory 220-47, California Institute of Technology, Pasadena, CA 91125, USA \\ ${ }^{5}$ Carnegie Observatories, 813 Santa Barbara Street, Pasadena, CA 91101, USA \\ ${ }^{6}$ Department of Astronomy, University of Michigan, 830 Dennison Bldg, Ann Arbor MI 48109 USA
}

Over the six years since the discovery ${ }^{1}$ of the $\gamma$-ray burst GRB 980425 , associated $^{2}$ with the nearby (distance, $\sim 40 \mathrm{Mpc}$ ) supernova 1998bw, astronomers have fiercely debated the nature of this event. Relative to bursts located at cosmological distances, (redshift, $z \sim 1$ ), GRB 980425 was under-luminous in $\gamma$-rays by three orders of magnitude. Radio calorimetry ${ }^{3,4}$ showed the explosion was sub-energetic by a factor of 10 . Here, we report observations of the radio and X-ray afterglow of the recent $z=0.105$ GRB $031203^{5-7}$ and demonstrate that it too is sub-energetic. Our result, when taken together with the low $\gamma$-ray luminosity ${ }^{7}$ suggest that GRB 031203 is the first cosmic analogue to GRB 980425 . We find no evidence that this event was a highly collimated explosion viewed off-axis. Like GRB 980425, GRB 031203 appears to be an intrinsically sub-energetic $\gamma$-burst. Such sub-energetic events have faint afterglows. Intensive follow-up of faint bursts with smooth $\gamma$-ray light curves ${ }^{8,9}$ (common to both GRBs 031203 and 980425) may enable us to reveal their expected large population.

Work Supported in part by the Department of Energy Contract DE-AC02-76SF00515

On 3 December 2003 at 22:01:28 UT, the INTEGRAL satellite detected ${ }^{5,7}$ a seemingly 
typical long-duration $(\Delta t \approx 20 \mathrm{sec}) \gamma$-ray burst. Within 6 hours, the Newton $X$-ray Multiple Mirror $(X M M)$ Observatory detected ${ }^{10,11}$ an X-ray source with flux $(2-10 \mathrm{keV}$

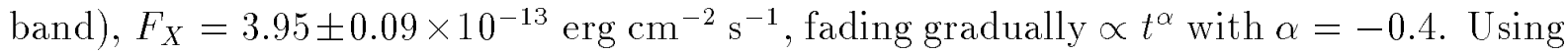
the Very Large Array (VLA), we discovered a radio source at $\alpha(\mathrm{J} 2000)=08^{\mathrm{h}} 02^{\mathrm{m}} 30.18^{\mathrm{s}}$ and $\delta(\mathrm{J} 2000)=-39^{\circ} 51^{\prime} 03.51^{\prime \prime}( \pm 0.1$ arcsec in each axis $)$, well within the 6 -arcsecond radius error circle of the $X M M$ source. A subsequent $X M M$ observation $^{12}$ confirmed the gradual decay of the X-ray source. From our analysis of the $X M M$ data we find the flux $\propto t^{-0.4}$ between the two epochs and the spectral flux density, $F_{\nu, X} \propto \nu^{\beta}$, is fit by $\beta=-0.81 \pm 0.05$ with an absorbing column density, $N_{\mathrm{H}}=6.2 \times 10^{21} \mathrm{~cm}^{-2}$. Taken together, the transient $\mathrm{X}$-ray and radio emission are suggestive of afterglow emission.

In addition to monitoring the afterglow in various radio bands (Table 1 and discussion below) we obtained an observation of the source with the Advanced CCD Imaging Spectrometer (ACIS) instrument aboard the Chandra X-ray Observatory (CXO). The CXO observations began on 22 January 2004 at 21:35 UT and lasted about 22 ksec. We detected a faint source, count rate in the $2-10 \mathrm{keV}$ band of $5.6 \times 10^{-4} \mathrm{~s}^{-1}$, at $\alpha(\mathrm{J} 2000)=08^{\mathrm{h}} 02^{\mathrm{m}} 30.159^{\mathrm{s}}$ and $\delta(\mathrm{J} 2000)=-39^{\circ} 51^{\prime} 03.51^{\prime \prime}( \pm 0.18$ arcsec in each axis $)$, precisely coincident with the VLA source. Using the XMM model parameters stated above we obtain $F_{X}=6.4 \times 10^{-15} \mathrm{erg} \mathrm{cm}^{-2} \mathrm{~s}^{-1}$, implying a faster decline $(\alpha=-1 \pm 0.1)$ between the second XMM and Chandra observations.

The primary interest in this burst is that the radio and $\mathrm{X}$-ray afterglow coincides at the sub-arcsecond leve ${ }^{13}$ with a nearby $(z=0.1055)$ galaxy, ${ }^{6}$ making it the nearest GRB with the exception of the peculiar GRB $980425 .^{2}$ At this redshift, the isotropic $\gamma$-ray energy release is $10^{2}$ times smaller ${ }^{7}$ than that of the nearest classical event GRB 030329 $(z=0.169)^{14}$ and yet a factor of $10^{2}$ larger ${ }^{1,2}$ than that of GRB 980425 .

The afterglow properties of GRB 031203 also appear to be intermediate between classical cosmological GRBs and GRB 980425: the isotropic X-ray luminosity of GRB 031203 at $t \approx 10$ hours is $L_{X}=9 \times 10^{42} \mathrm{erg} \mathrm{cm}^{-2} \mathrm{~s}^{-1}$, nearly $10^{3}$ times fainter than that observed $^{15}$ for classical GRBs but a factor of $10^{2}$ brighter $^{1}$ than that of GRB 980425 . In the centimetre band, the peak luminosity is $L_{\nu, 8.5 \mathrm{GHz}} \approx 10^{29} \mathrm{erg} \mathrm{s}^{-1} \mathrm{~Hz}^{-1}$, fainter ${ }^{16}$ by a factor of $10^{2}$ than that of most radio afterglows but comparable ${ }^{3}$ to that of GRB 980425 . Since $L_{X}$ and peak radio luminosity of an afterglow can be used ${ }^{17,15}$ as rough proxies for 
the afterglow energy, the data suggest that GRBs 031203 and 980425 are sub-energetic in comparison with classical GRBs.

As a next step, we applied the simplest afterglow model ${ }^{18,19}$ (a spherical relativistic blastwave shocking a constant density circumburst medium and accelerating relativistic electrons; the afterglow emission arises from synchrotron emission of shocked electrons) to the afterglow data and obtain a satisfactory fit (Figure 1). On the timescales best probed by the radio data - days to months - we see no evidence for a collimated (jet) geometry commonly $\operatorname{seen}^{20}$ in the afterglows of cosmological GRBs.

From our modeling we confirm that the blast wave is sub-energetic, finding an inferred afterglow energy of $E_{\mathrm{AG}} \approx 1.7 \times 10^{49} \mathrm{erg}$. The circumburst particle density $n \approx 0.6 \mathrm{~cm}^{-3}$, is not atypical of that inferred ${ }^{17}$ for other GRBs. The blastwave is expected to become ${ }^{21}$ non-relativistic on a timescale, $t_{\mathrm{NR}} \approx 34\left(E_{\mathrm{AG}, 50} / n_{0}\right)^{1 / 3} \mathrm{~d}$, where we adopt the notation $q \equiv 10^{x} q_{x}$. The observational signatures ${ }^{22}$ of this transition, a steeper decay of the spectral peak frequency $\left(\nu_{m} \propto t^{-1.5} \rightarrow t^{-3}\right)$ and an increase in the spectral peak flux $\left(F_{\nu_{m}} \propto t^{0} \rightarrow t^{3 / 5}\right)$ are consistent with the data (Figure 1$)$.

Here we use $E_{\mathrm{AG}}$ to denote the kinetic energy remaining in the blast wave after the prompt $\gamma$-ray energy release. In turn, the $\gamma$-ray emission arises from ultra-relativistic (bulk Lorentz factor, $\Gamma \gtrsim 100$ ) ejecta within the blastwave. Thus, a more complete picture of the explosion energy is visualized through a two-dimensional plot of $E_{\text {prompt }}$, the beaming-corrected prompt energy release versus $E_{\mathrm{AG}}$ (Figure 2).

The two nearest events, GRBs 031203 and 980425, are clearly sub-energetic outliers in Figure 2. Furthermore, we draw the reader's attention to several additional similarities: GRBs 031203 and 980425 (1) show no evidence for jets, ${ }^{3}(2)$ possess simple $\gamma$-ray light curves $^{1,7}$; and with respect to cosmological ("classical") bursts the two events (3) violate the $E_{\text {prompt }}-E_{\text {peak }}$ relation ${ }^{23}$ and (4) are outliers in the luminosity - spectral lag relation. ${ }^{9}$ This discussion motivates the question: How are these two events related to cosmological GRBs?

It has been suggested (e.g. ref 24) that all GRB explosions have the same energetics and explosion geometry. In this framework, sub-energetic bursts are simply events viewed away from the jet axis. Such bursts should have a soft $E_{\text {peak }}$ and also exhibit a rise in the inferred $E_{\mathrm{AG}}$ as shocked ejecta eventually come into our line of sight. For GRB 031203, 
$E_{\text {peak }}>190 \mathrm{keV}$ (ref. 7), comparable to cosmological GRBs for which we have observational evidence favoring an on-axis viewing angle. Moreover, we see no evidence for an increase in $E_{\mathrm{AG}}$ during the timescale of the radio observations ( $\sim 150$ days). Similarly, there is no evidence that $E_{\mathrm{AG}}$ is increasing for GRB 980425 despite dedicated radio monitoring $^{25}$ of the source since 1998. With no indication of being off-axis explosions, we presently conclude that GRBs 031203 and 980425 are intrinsically sub-energetic events.

Astronomers have had to wait six years to discover a sub-energetic event similar to GRB 980425, despite a large population (as implied by their proximity). The bulk of the population has escaped our attention due to their faint $\gamma$-ray and afterglow emission which challenge our current detection limits. The Swift satellite mission, with its higher $\gamma$-ray sensitivity (compared to current missions) and improved localization capability (enabling rapid identification of afterglow counterparts) is expected to revolutionize our understanding of cosmic explosions.

Received 4 August 2004; Accepted draft.

1. Pian, E., Amati, L., Antonelli, L. A., Butler, R. C., Costa, E. et al. BEPPOSAX Observations of GRB 980425: Detection of the Prompt Event and Monitoring of the Error Box. Astrophys. J. 536, 778-787 June 2000.

2. Galama, T. J., Vreeswijk, P. M., van Paradijs, J., Kouveliotou, C., Augusteijn, T. et al. An unusual supernova in the error box of the gamma-ray burst of 25 April 1998. Nature 395, $670-672(1998)$.

3. Kulkarni, S. R., Frail, D. A., Wieringa, M. H., Ekers, R. D., Sadler, E. M. et al. Radio emission from the unusual supernova $1998 \mathrm{bw}$ and its association with the gamma-ray burst of 25 April 1998. Nature 395, 663-669 (1998).

4. Li, Z. \& Chevalier, R. A. Radio Supernova SN 1998BW and Its Relation to GRB 980425. Astrophys. J. 526, 716-726 December 1999.

5. Gotz, D., Mereghetti, S., Beck, M., Borkowski, J. \& Mowlavi, N. GRB 031203: a long GRB detected with INTEGRAL. (2003).

6. Prochaska, J. X., Bloom, J. S., Chen, H., Hurley, K. C., Melbourne, J. et al. The Host Galaxy of GRB 031203: Implications of its low metallicity, low redshift, and starburst nature. submitted to ApJ February 2004. 
7. Sazonov, S. Y., Lutovinov, A. A. \& Sunyaev, R. A. An apparently normal gamma-ray burst with an unusally low luminosity. submitted to Nature (2004).

8. Bloom, J. S., Kulkarni, S. R., Harrison, F., Prince, T., Phinney, E. S. et al. Expected Characteristics of the Subclass of Supernova Gamma-Ray Bursts. Astrophys. J. 506, L105L108 October 1998.

9. Norris, J. P. Implications of the Lag-Luminosity Relationship for Unified Gamma-Ray Burst Paradigms. Astrophys. J. 579, 386-403 November 2002.

10. Santos-Lleo, M., Calderon, P. \& Gotz, D. GRB 031203 XMM-newton observation. (2003).

11. Watson, D., Hjorth, J., Levan, A., Jakobsson, P., O’Brien, P. T. et al. A very low luminosity X-ray flash: XMM-Newton observations of GRB 031203. submitted to ApJL (2004).

12. Rodriguez-Pascual, P., Santos-Lleo, M., Gonzalez-Riestra, R., Schartel, N. \& Altieri, B. XMM-Newton observation of GRB031203. (2003).

13. Gal-Yam, A., Moon, D.-S., Fox, D. B., Soderberg, A. M., Kulkarni, S. R. et al. The near infra-red light curve of sn 20031w, associated with grb 031203. submitted to ApJ (2004).

14. Vanderspek, R. et al. Hete observations of the gamma-ray burst grb030329: Evidence for an underlying soft x-ray component. astro-ph/0401311 (2004).

15. Berger, E., Kulkarni, S. R. \& Frail, D. A. A Standard Kinetic Energy Reservoir in GammaRay Burst Afterglows. Astrophys. J. 590, 379-385 June 2003.

16. Frail, D. A., Kulkarni, S. R., Berger, E. \& Wieringa, M. H. A Complete Catalog of Radio Afterglows: The First Five Years. Astron. J. 125, 2299-2306 May 2003.

17. Panaitescu, A. \& Kumar, P. Properties of Relativistic Jets in Gamma-Ray Burst Afterglows. Astrophys. J. 571, 779-789 June 2002.

18. Sari, R., Piran, T. \& Narayan, R. Spectra and Light Curves of Gamma-Ray Burst Afterglows. Astrophys. J. 497, L17+ April 1998.

19. Granot, J. \& Sari, R. The Shape of Spectral Breaks in Gamma-Ray Burst Afterglows. Astrophys. J. 568, 820-829 April 2002.

20. Frail, D. A., Kulkarni, S. R., Sari, R., Djorgovski, S. G., Bloom, J. S. et al. Beaming in Gamma-Ray Bursts: Evidence for a Standard Energy Reservoir. Astrophys. J. 562, L55L58 November 2001. 
21. Waxman, E. The nature of GRB980425 and the search for off-axis GRB signatures in nearby type Ib/c supernovae emission. submitted to ApJ October 2003.

22. Frail, D. A., Waxman, E. \& Kulkarni, S. R. A 450 Day Light Curve of the Radio Afterglow of GRB 970508: Fireball Calorimetry. Astrophys. J. 537, 191-204 July 2000.

23. Amati, L., Frontera, F., Tavani, M., in’t Zand, J. J. M., Antonelli, A. et al. Intrinsic spectra and energetics of BeppoSAX Gamma-Ray Bursts with known redshifts. 390, 81-89 July 2002.

24. Nakamura, T., Mazzali, P. A., Nomoto, K. \& Iwamoto, K. Light Curve and Spectral Models for the Hypernova SN 1998BW Associated with GRB 980425. Astrophys. J. 550, 991-999 April 2001.

25. Soderberg, A. M., Frail, D. A. \& Wieringa, M. A. Severe constraints on off-axis grb jets in type ibc supernovae from late-time radio observations. astro-ph/0402163 (2004).

26. Bloom, J. S., Frail, D. A. \& Kulkarni, S. R. Gamma-Ray Burst Energetics and the GammaRay Burst Hubble Diagram: Promises and Limitations. Astrophys. J. 594, 674-683 September 2003.

27. Berger, E., Kulkarni, S. R., Pooley, G., Frail, D. A., McIntyre, V. et al. A common origin for cosmic explosions inferred from calorimetry of GRB030329. Nature 426, 154-157 November 2003.

28. Soderberg, A. M., Kulkarni, S. R., Berger, E., Fox, D. B., Price, P. A. et al. A Redshift Determination for XRF 020903: First Spectroscopic Observations of an X-Ray Flash. astroph/0311050 November 2003.

29. Berger, E., Kulkarni, S. R., Frail, D. A. \& Soderberg, A. M. A Radio Survey of Type Ib and Ic Supernovae: Searching for Engine-driven Supernovae. Astrophys. J. 599, 408-418 December 2003.

Correspondence should be addressed to A. M. Soderberg (e-mail:ams@astro.caltech.edu).

\section{Acknowledgements}

GRB research at Caltech is supported in part by funds from NSF and NASA. We are, as always, indebted to Scott Barthelmy and the GCN. The VLA is operated by the National Radio Astronomy Observatory, a facility of the National Science Foundation operated 
under cooperative agreement by Associated Universities, Inc.. AMS is supported by an NSF Graduate Research Fellowship. AG acknowledges support by NASA through a Hubble Fellowship grant. 


\begin{tabular}{lccccc}
\hline \hline $\begin{array}{l}\text { Epoch } \\
(\mathrm{UT})\end{array}$ & $\begin{array}{c}\Delta t \\
(\text { days })\end{array}$ & $\begin{array}{c}F_{1.43} \\
(\mathrm{mJy})\end{array}$ & $\begin{array}{c}F_{4.86} \\
(\mathrm{mJy})\end{array}$ & $\begin{array}{c}F_{8.46} \\
(\mathrm{mJy})\end{array}$ & $\begin{array}{c}F_{22.5} \\
(\mathrm{mJy})\end{array}$ \\
\hline 2003 Dec 5.52 & 1.60 & - & - & $0.540 \pm 0.062$ & - \\
2003 Dec 7.52 & 3.60 & - & - & $0.249 \pm 0.043$ & - \\
2003 Dec 8.35 & 4.43 & - & $0.393 \pm 0.060$ & $0.053 \pm 0.052$ & - \\
2003 Dec 12.38 & 8.46 & - & - & $0.280 \pm 0.049$ & - \\
2003 Dec 15.37 & 11.45 & - & - & $0.304 \pm 0.042$ & - \\
2003 Dec 17.38 & 13.46 & - & $0.520 \pm 0.050$ & $0.448 \pm 0.039$ & $0.483 \pm 0.083$ \\
2003 Dec 21.35 & 17.43 & - & - & $0.457 \pm 0.041$ & - \\
2003 Dec 23.37 & 19.45 & - & - & $0.811 \pm 0.040$ & - \\
2003 Dec 26.40 & 22.48 & - & $0.583 \pm 0.054$ & $0.467 \pm 0.046$ & - \\
2003 Dec 31.33 & 27.41 & - & - & $0.675 \pm 0.045$ & - \\
2004 Jan 4.33 & 31.41 & - & $0.728 \pm 0.055$ & $0.459 \pm 0.047$ & - \\
2004 Jan 8.26 & 35.34 & - & $0.624 \pm 0.050$ & $0.308 \pm 0.043$ & - \\
2004 Jan 12.29 & 39.37 & $1.011 \pm 0.113$ & $0.598 \pm 0.063$ & $0.647 \pm 0.045$ & - \\
2004 Jan 15.35 & 42.43 & $0.689 \pm 0.136$ & $0.749 \pm 0.063$ & $0.664 \pm 0.061$ & - \\
2004 Jan 25.24 & 52.32 & $0.710 \pm 0.082$ & - & $0.450 \pm 0.044$ & - \\
2004 Jan 26.34 & 53.42 & - & $0.556 \pm 0.058$ & - & - \\
2004 Feb 7.24 & 65.32 & $0.937 \pm 0.112$ & $0.751 \pm 0.045$ & $0.533 \pm 0.028$ & $0.273 \pm 0.066$ \\
2004 Feb 15.22 & 73.30 & $0.756 \pm 0.147$ & $0.576 \pm 0.050$ & $0.517 \pm 0.042$ & - \\
2004 Feb 28.13 & 86.21 & - & - & $0.517 \pm 0.047$ & $0 \pm 0.114$ \\
2004 Mar 6.17 & 93.25 & $0.631 \pm 0.091$ & $0.522 \pm 0.058$ & $0.304 \pm 0.046$ & - \\
2004 Mar 23.13 & 110.21 & $0.787 \pm 0.169$ & $0.593 \pm 0.062$ & $0.432 \pm 0.042$ & - \\
2004 Apr 19.07 & 137.15 & - & - & $0.426 \pm 0.037$ & -
\end{tabular}

Table 1. Radio observations made with the Very Large Array (VLA). Observations commenced on 5 December 2003 UT. For all observations we used the standard continuum mode with $2 \times 50$ $\mathrm{MHz}$ bands. At $22.5 \mathrm{GHz}$ we used referenced pointing scans to correct for the systematic $10-20$ arcsec pointing errors of the VLA antennas. We used the extra-galactic sources 3C 147 $(\mathrm{J} 0542+498)$ and $3 \mathrm{C} 286(\mathrm{~J} 1331+305)$ for flux calibration, while the phase was monitored using J0828-375. The data were reduced and analyzed using the Astronomical Image Processing System. The flux density and uncertainty were measured from the resulting maps by fitting a Gaussian model to the afterglow emission. 
Figure 1: Radio lightcurves of the afterglow of GRB 031203. All measurements are summarized in Table 1 and include $1-\sigma$ error bars. The solid lines are models of synchrotron (afterglow) emission from spherical ejecta expanding into a uniform circumburst medium. ${ }^{19}$ The models include a contribution from the host galaxy, which is well-fit by $F_{\text {host }} \approx 0.4(\nu / 1.4 \mathrm{GHz})^{-0.6} \mathrm{mJy}$ (dashed lines) and is consistent with the star formation rate inferred ${ }^{6}$ from optical spectroscopy of the host. In applying the models, the X-ray observations a re considered upper-limits since they are most likely dominated by (non-synchrotron) emission arising from the associated supernova SN 20031w, as evidenced by the unusually slow flux decay at early time and the flat spectral index $\left(F_{\nu, X} \propto t^{-0.4} \nu^{-0.8}\right.$ as opposed to $\propto t^{-1} \nu^{-1.3}$ for GRBs). This was also the case for the X-ray emission $^{1}$ of GRB $980425 / \mathrm{SN} 1998 \mathrm{bw}\left(F_{\nu, X} \propto t^{-0.2} \nu^{-1}\right)$. For our best-fit model, we find $\chi_{r}^{2}=8.9$ (38 degrees of freedom), dominated by interstellar scintillation. The blastwave transitions to the non-relativistic regime at $t_{N R} \approx 23 \mathrm{~d}$. From the derived synchrotron parameters $($ at $t=1 \mathrm{~d}$ ): $\nu_{a} \approx 3.2 \times 10^{8} \mathrm{~Hz}, \nu_{m} \approx 3.6 \times 10^{12} \mathrm{~Hz}$ and $F_{\nu_{a}} \approx 0.04 \mathrm{mJy}$ we find an isotropic afterglow energy, $E_{\mathrm{AG}, \text { iso }} \approx 1.7 \times 10^{49} \nu_{c, 15.5}^{1 / 4} \mathrm{erg}$, a circumburst density $n \approx 0.6 \nu_{c, 15.5}^{3 / 4} \mathrm{~cm}^{-3}$ and the fractions of energy in the relativistic electrons (energy distribution $N(\gamma) \propto \gamma^{-p}$ with $p \approx 2.6$ ) and magnetic field of $\epsilon_{e} \approx 0.4 \nu_{c, 15.5}^{1 / 4}$ and $\epsilon_{B} \approx 0.2 \nu_{c, 15.5}^{-5 / 4}$, respectively. Here, $\nu_{c}=3 \times 10^{15} \nu_{c, 15.5}$ is the synchrotron cooling frequency which is roughly constrained by the (non-synchrotron) SN 20031w X-ray emission. Extrapolation of the synchrotron model beyond $\nu_{c}$ underestimates the observed X-ray flux by a factor of $\lesssim 10$ which is comparable to the discrepancy for SN 1998 bw (found by extrapolating the radio model by Li \& Chevalier $^{4}\left(p=2.5, \epsilon_{B}=10^{-3}\right)$ beyond $\nu_{c}$ and comparing with the $\mathrm{X}$-ray data ${ }^{1}$ at $t \sim 12$ days).

Figure 2: Two-dimensional energy plot for cosmic explosions. The energy in the prompt emission, $E_{\text {prompt }}$, and in the afterglow, $E_{\mathrm{AG}}$, have been corrected ${ }^{20,26,27}$ for beaming based on the jet-break time observed for each burst, except in the cases of GRB $980425,{ }^{4,3}$ XRF $020903^{28}$ and GRB 031203 for which there is no evidence for a collimated outflow. For these three cases we plot the isotropic values of $E_{\text {prompt }}$ and $E_{\mathrm{AG}}$ and use an arrow to indicate they represent upper limits on both axes. The arcs mark lines of constant $E_{\mathrm{prompt}}+E_{\mathrm{AG}}$ as a guide to the reader. Most cosmological GRBs tend to cluster $^{27}$ around $E_{\text {prompt }}+E_{\mathrm{AG}} \approx 2 \times 10^{51}$ erg while GRBs 031203 and 980425 , the nearest two bursts in the sample, are clearly sub-energetic. With the exception of SN 1998bw, associated with GRB 980425, there are no local Ibc supernovae with 
$10 \quad$ Soderberg et al.

detected $\gamma$-ray emission, however the kinetic energy in the ejecta (excluding the photospheric energy yield), is generally found ${ }^{29}$ to be $E_{\mathrm{AG}} \lesssim 3 \times 10^{48} \mathrm{erg}$ (bottom left corner). Histograms of $E_{\mathrm{AG}}$ and $E_{\text {prompt }}$ are shown in the bottom and side panels, respectively, for cosmological GRBs and local Ibc SNe. The striped energy bins show the locations of GRBs 980425 and 031203. 


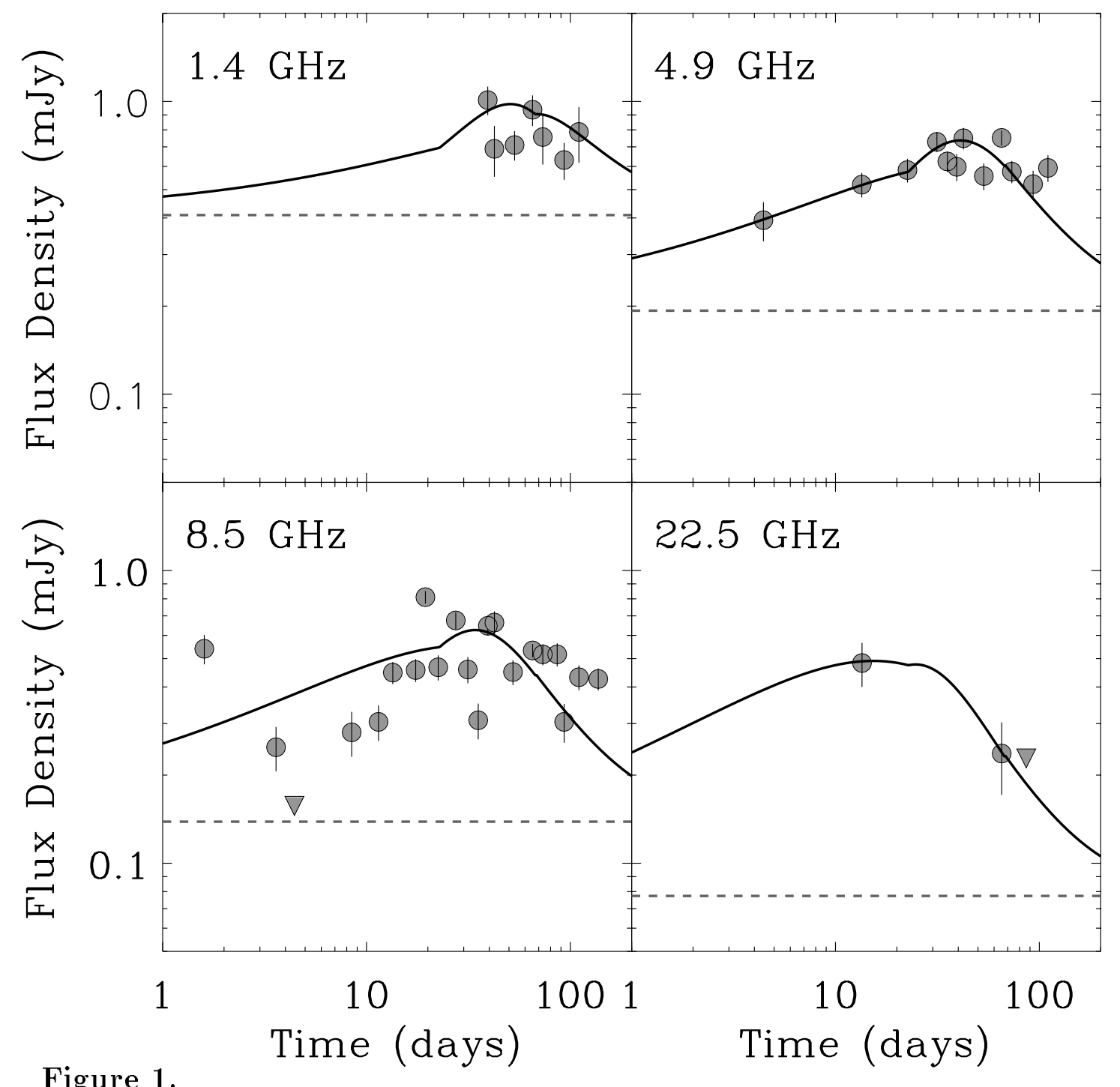

Figure 1. 


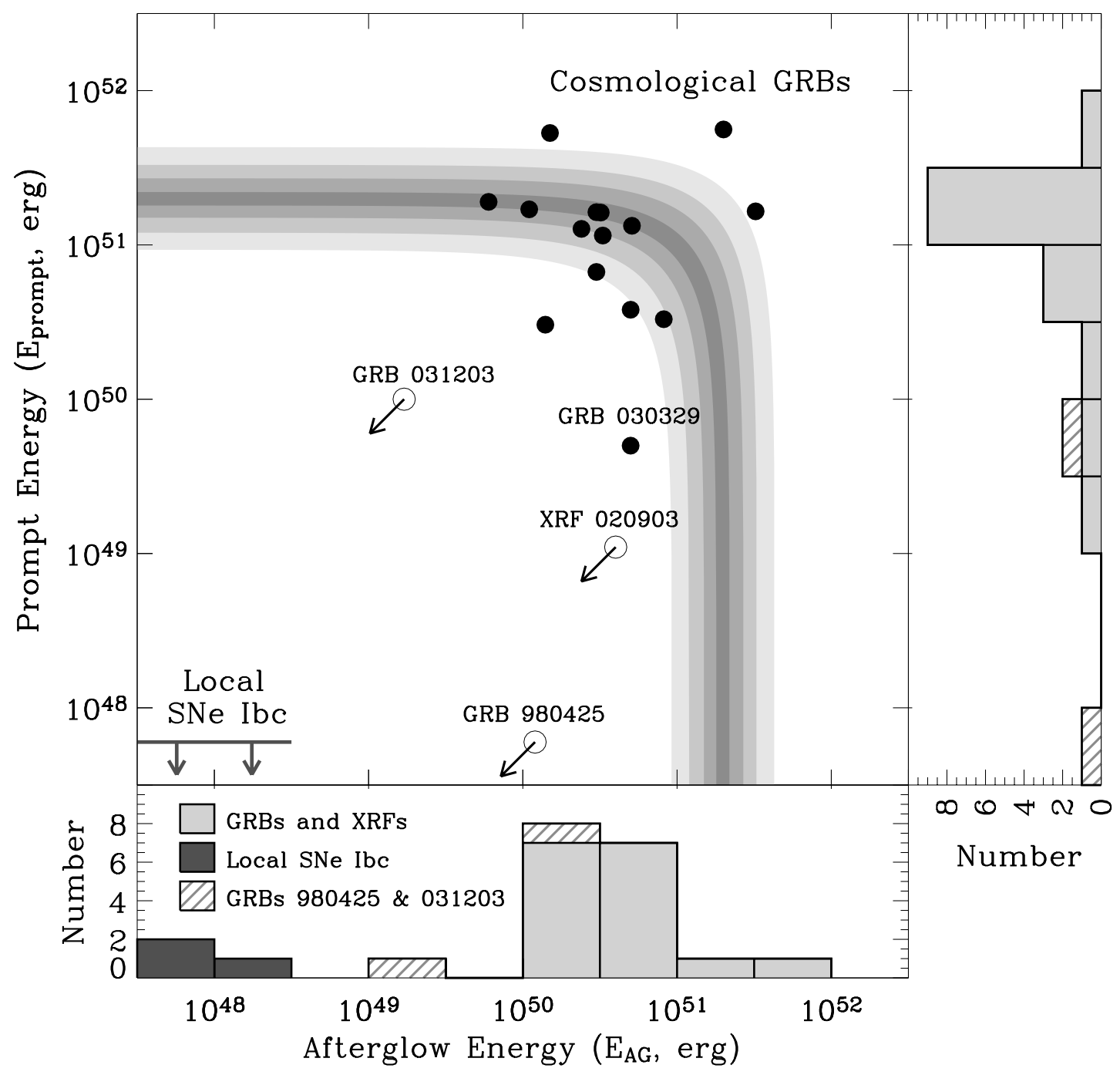

Figure 2. 